\title{
A COMPLETE SET OF UNITARY INVARIANTS FOR $3 \times 3$ COMPLEX MATRICES
}

\author{
BY \\ CARL PEARCY
}

1. Introduction. The question of finding a complete set of unitary invariants for $n \times n$ matrices, for a long time considered to be an unsolved problem, was given a more or less satisfactory solution by Specht [4]. Consider the free multiplicative semi-group $W$ generated by the symbols $x$ and $y$, and denote words in $W$ by $w(x, y)$. In [4], Specht showed that the collection of traces

$$
\left\{\sigma\left[w\left(A, A^{*}\right)\right] / w(x, y) \in W\right\}
$$

is a complete set of unitary invariants for any $n \times n$ complex matrix $A$. The author was able to improve this result by demonstrating in [3] that for $n$ fixed but arbitrary, there is always a subset of the above collection containing fewer than $2^{4 n^{2}}$ traces which serves as a complete set of unitary invariants for $n \times n$ matrices. It is of interest to discover the sharpness of the above upper bound on the number of traces needed, and thus it was desired to compare the estimate $2^{4 n^{2}}$ with any known results. Unfortunately, even though there is a vast literature on the subject, to the author's knowledge no solution to the problem even for small $n$ has been given, except in the case $n=2$. Murnaghan [1] showed that for $n=2$, the traces $\sigma(A), \sigma\left(A^{2}\right)$, and $\sigma\left(A^{*} A\right)$, form a complete set of invariants, and he began a study of the case $n=3$. He did not carry his analysis far enough to solve the problem however and, in fact, his result that six traces suffice in the case that the eigenvalues are distinct is incorrect, as will be shown by an example in $\S 3$.

It is the purpose of this paper to complete the analysis of the case $n=3$ by demonstrating that there is a collection of nine traces which suffice in this situation.

2. The idea is to treat the different eigenvalue possibilities as separate cases, obtain what turns out to be a canonical form under unitary transformations, and then show that two matrices in this form which possess the same nine appropriate traces are equal. We begin with four lemmas of an elementary nature. Details of the proofs of these lemmas can be found in [1]. For the triangular matrix

$$
\left(\begin{array}{lll}
a_{11} & a_{12} & a_{13} \\
0 & a_{22} & a_{23} \\
0 & 0 & a_{33}
\end{array}\right)
$$

Received by the editors August 30, 1961. 
we introduce the notation $\operatorname{Tri}\left(a_{11}, a_{22}, a_{33}, a_{12}, a_{23}, a_{13}\right)$.

Leмма 2.1. If $A$ is any $3 \times 3$ complex matrix having one eigenvalue a of (algebraic) multiplicity 3 , then $A$ is unitarily equivalent to a matrix $\operatorname{Tri}(a, a, a, d, g, f)$ where

(1) $d, g \geqq 0$,

(2) $d=0$ implies $g=0$,

(3) $g=0$ implies both $d=0$ and $f \geqq 0$.

Lemмa 2.2. If $A$ has one eigenvalue $b$ of multiplicity 2 and another $a$ of multiplicity 1 , then $A$ is unitarily equivalent to a matrix $\operatorname{Tri}(b, b, a, d, g, f)$ where

(1) $d, g \geqq 0$,

(2) $d=0$ implies $g=0$,

(3) $g=0$ implies $f \geqq 0$.

Lemмa 2.3. If $A$ has distinct eigenvalues and $(a, b, c)$ is any desired ordering of these eigenvalues, then $A$ is unitarily equivalent to a matrix $\operatorname{Tri}(a, b, c, d, g, f)$ where

(1) $d, g \geqq 0$,

(2) $d=0$ or $g=0$ implies $f \geqq 0$.

Lemma 2.4. Suppose $A_{1}$ and $A_{2}$ are the matrices $A_{i}=\operatorname{Tri}\left(e_{1}, e_{2}, e_{3}, d_{i}, g_{i}, f_{i}\right)$ where $d_{i}, g_{i} \geqq 0$, and suppose the six corresponding traces $\sigma\left(A_{i}\right), \sigma\left(A_{i}^{2}\right), \sigma\left(A_{i}^{3}\right)$, $\sigma\left(A_{i}^{*} A_{i}\right), \sigma\left(A_{i}^{*} A_{i}^{2}\right)$, and $\sigma\left(A_{i}^{* 2} A_{i}^{2}\right)$ are equal for $i=1,2$. Then the three corresponding numbers

$$
\begin{gathered}
d_{i}^{2}+g_{i}^{2}+\left|f_{i}\right|^{2}, \\
\left|e_{3}-e_{2}\right|^{2} d_{i}^{2}+\left|e_{2}-e_{1}\right|^{2} g_{i}^{2}+d_{i}^{2} g_{i}^{2} \\
\left(e_{3}-e_{2}\right) d_{i}^{2}+\left(e_{1}-e_{2}\right) g_{i}^{2}-d_{i} g_{i} f_{i}^{*}
\end{gathered}
$$

are equal for $i=1,2$.

Now consider the sequence $T(A)$ of nine numbers $\left\{\sigma(A), \sigma\left(A^{2}\right), \sigma\left(A^{3}\right)\right.$, $\left.\sigma\left(A^{*} A\right), \sigma\left(A^{*} A^{2}\right), \sigma\left(A^{* 2} A^{2}\right), \sigma\left(A^{*} A A^{*} A\right), \sigma\left(A^{*} A^{2} A^{*} A\right), \sigma\left(A^{*} A^{2} A^{* 2} A\right)\right\}$. If $A$ and $B$ are matrices, then $T(A)=T(B)$ means that each pair of corresponding numbers are equal. As mentioned above, our plan is to show that if $A$ and $B$ are both of the form of Lemma 2.1 (or Lemma 2.2, Lemma 2.3) and $T(A)=T(B)$, then $A=B$. To prove $A=B$, it suffices to prove that $A-\lambda I$ $=B-\lambda I$ where $I$ is the identity matrix and $\lambda$ is any scalar, and the matrices $A-\lambda I$ and $B-\lambda I$ are sometimes easier to calculate with. Thus we need

Lemma 2.5. If $A$ and $B$ are $3 \times 3$ matrices such that $T(A)=T(B)$, and $\lambda$ is any complex number, then $T(A-\lambda I)=T(B-\lambda I)$.

Proof. The proof uses the facts that the trace function is linear, the trace of any commutator is zero, and $\sigma\left(A^{*}\right)=[\sigma(A)]^{*}$. It follows from the equalities 
$\sigma\left(A^{i}\right)=\sigma\left(B^{i}\right), i=1,2,3$, that $A$ and $B$ have the same characteristic equation, and this in turn implies that $\sigma\left(A^{3} A^{*}\right)=\sigma\left(B^{3} B^{*}\right)$ and $\sigma\left(A^{3} A^{* 2}\right)=\sigma\left(B^{3} B^{* 2}\right)$. [Expand $A^{3}$ as $\alpha A^{2}+\delta A+\gamma I$, and similarly for $B$.] Now any of the nine words whose trace appears in $T(A-\lambda I)$ can be expanded into a linear combination of words $w_{\beta}\left(A, A^{*}\right)$ such that for each $\beta, \sigma\left[w_{\beta}\left(A, A^{*}\right)\right]=\sigma\left[w_{\beta}\left(B, B^{*}\right)\right]$, and the result follows by recombining the $w_{\beta}\left(B, B^{*}\right)$ into the appropriate word in $B-\lambda I$. We make one sample calculation and omit the rest.

$$
\begin{aligned}
\sigma & {\left[(A-\lambda I)^{* 2}(A-\lambda I)\right] } \\
& =\sigma\left(A^{* 2} A\right)-\lambda \sigma\left(A^{* 2}\right)-2 \lambda^{*} \sigma\left(A^{*} A\right)+2|\lambda|{ }^{2} \sigma\left(A^{*}\right)+\lambda^{* 2} \sigma(A)-\lambda^{*}|\lambda|{ }^{2} \sigma(I) \\
& =\sigma\left(B^{* 2} B\right)-\lambda \sigma\left(B^{* 2}\right)-2 \lambda^{*} \sigma\left(B^{*} B\right)+2|\lambda|{ }^{2} \sigma\left(B^{*}\right)+\lambda^{* 2} \sigma(B)-\lambda^{*}|\lambda|{ }^{2} \sigma(I) \\
& =\sigma\left[(B-\lambda I)^{* 2}(B-\lambda I)\right] .
\end{aligned}
$$

Lemma 2.6. Suppose $A_{i}, i=1,2$, are the matrices $\operatorname{Tri}\left(a, a, a, d_{i}, g_{i}, f_{i}\right)$ each having properties (1), (2), and (3) of Lemma 2.1 and satisfying $T\left(A_{1}\right)$ $=T\left(A_{2}\right)$. Then $A_{1}=A_{2}$.

Proof. From Lemma 2.5 we have $T\left(A_{1}-a I\right)=T\left(A_{2}-a I\right)$, and it results from the $4 \mathrm{th}, 5 \mathrm{th}, 6 \mathrm{th}$, and 9 th of the nine resulting equalities that

$$
\begin{aligned}
d_{1}^{2}+g_{1}^{2}+\left|f_{1}\right|^{2} & =d_{2}^{2}+g_{2}^{2}+\left|f_{2}\right|^{2}, \\
d_{1} g_{1} f_{1}^{*} & =d_{2} g_{2} f_{2}^{*}, \\
d_{1}^{2} g_{1}^{2} & =d_{2}^{2} g_{2}^{2} \\
d_{1}^{2} g_{1}^{2}\left(d_{1}^{2}+\left|f_{1}\right|^{2}\right) & =d_{2}^{2} g_{2}^{2}\left(d_{2}^{2}+\left|f_{2}\right|^{2}\right) .
\end{aligned}
$$

Since $d_{1}, g_{1} \geqq 0$ it follows from (3) that $d_{1} g_{1}=d_{2} g_{2}$. If $d_{1} g_{1} \neq 0$, then division of (2) yields $f_{1}=f_{2}$ and from (4) we get $d_{1}=d_{2}$. Then (1) yields $g_{1}=g_{2}$ and thus $A_{1}=A_{2}$. If $d_{1} g_{1}=0$, then by hypothesis $d_{1}=g_{1}=d_{2}=g_{2}=0$ and $f_{1}, f_{2} \geqq 0$. Taking square roots in (1) completes the argument.

Lemma 2.7. Suppose $A_{i}, i=1,2$, are the matrices $\operatorname{Tri}\left(b, b, a, d_{i}, g_{i}, f_{i}\right)$ each having properties (1), (2), and (3) of Lemma 2.2 and also the property that $T\left(A_{1}\right)=T\left(A_{2}\right)$. Then $A_{1}=A_{2}$.

Proof. Denote $a-b$ by $k$. From Lemma 2.5 we have $T\left(A_{1}-b I\right)$ $=T\left(A_{2}-b I\right)$, and it follows by combining the 5 th, 6 th, and 9 th of the resulting equations that

$$
\begin{gathered}
d_{1}^{2} g_{1}^{2}\left|d_{1} g_{1}+f_{1} k\right|^{2}=d_{2}^{2} g_{2}^{2}\left|d_{2} g_{2}+f_{2} k\right|^{2}, \\
d_{1}^{2}\left|d_{1} g_{1}+f_{1} k\right|^{2}=d_{2}^{2}\left|d_{2} g_{2}+f_{2} k\right|^{2} .
\end{gathered}
$$

Also, after the appropriate change of notation, we have from Lemma 2.4

$$
\left(|k|^{2}+g_{1}^{2}\right) d_{1}^{2}=\left(|k|^{2}+g_{2}^{2}\right) d_{2}^{2},
$$




$$
\begin{gathered}
d_{1}^{2}+g_{1}^{2}+\left|f_{1}\right|^{2}=d_{2}^{2}+g_{2}^{2}+\left|f_{2}\right|^{2}, \\
k d_{1}^{2}-d_{1} g_{1} f_{1}^{*}=k d_{2}^{2}-d_{2} g_{2} f_{2}^{*} .
\end{gathered}
$$

Now if $d_{1}^{2}\left|d_{1} g_{1}+f_{1} k\right|^{2} \neq 0$, then dividing (1) by (2) gives $g_{1}=g_{2}$. Dividing (3) by $|k|^{2}+g_{i}^{2}$, which is nonzero, yields $d_{1}=d_{2}$. If $g_{i} \neq 0$, then (5) yields $f_{1}=f_{2}$. If $g_{i}=0$, then by hypothesis $f_{i} \geqq 0$, and (4) yields $f_{1}=f_{2}$. Turning to the case $d_{1}^{2}\left|d_{1} g_{1}+f_{1} k\right|^{2}=0$, there are two possibilities. If $d_{1}=0$, it follows from (3) that $d_{2}=0$. By hypothesis then $g_{1}=g_{2}=0, f_{i} \geqq 0$, and $f_{1}=f_{2}$ follows from (4). If $d_{1} g_{1}+f_{1} k=0, d_{1} \neq 0$, then $d_{2} \neq 0$ by (3), so $\left|d_{2} g_{2}+f_{2} k\right|=0$. Thus $\left|f_{i}\right|^{2}=d_{i}^{2} g_{i}^{2} /|k|^{2}$ for $i=1,2$, and substitution in (4) gives

$$
\left(d_{1}^{2}+g_{1}^{2}\right)|k|^{2}+d_{1}^{2} g_{1}^{2}=\left(d_{2}^{2}+g_{2}^{2}\right)|k|^{2}+d_{2}^{2} g_{2}^{2} \text {. }
$$

Subtraction of this equation from (3) yields $|k|^{2} g_{1}^{2}=|k|^{2} g_{2}^{2}$ and thus $g_{1}=g_{2}$. Then (3) gives $d_{1}=d_{2}$, and $f_{1}=f_{2}$ follows easily.

Lemma 2.8. Suppose $A_{i}, i=1,2$, are the matrices Tri $\left(a, b, c, d_{i}, g_{i}, f_{i}\right)$ each having properties (1), (2), and (3) of Lemma 2.3 and also satisfying $T\left(A_{1}\right)$ $=T\left(A_{2}\right)$. Then $A_{1}=A_{2}$.

Proof. Write $b-a=k_{1}, c-a=k_{2}$, and $\left(A_{i}-a I\right)=C_{i}$. Calculation shows that $C_{i}^{*}\left(C_{i}^{2}-k_{1} C_{i}\right)$ is the matrix $B_{i}=\operatorname{Tri}\left(0,0, p_{i}, 0, q_{i}, 0\right)$ where

and

$$
p_{i}=g_{i}\left(d_{i} f_{i}^{*}+g_{i} k_{1}\right)+\left(k_{2}-k_{1}\right)\left(\left|f_{i}\right|^{2}+g_{i}^{2}+\left|k_{2}\right|^{2}\right)
$$

$$
q_{i}=g_{i}\left(d_{i}^{2}+\left|k_{1}\right|^{2}\right)+\left(k_{2}-k_{1}\right)\left(f_{i} d_{i}+k_{1}^{*} g_{i}\right) .
$$

We first observe that $C_{i}^{2}-k_{1} C_{i} \neq 0$, since the minimal polynomial of $C_{i}$ is of degree three. It follows that $B_{i} \neq 0$, since $B_{i}=0$ implies that $\left(C_{i}^{2}-k_{1} C_{i}\right)^{*}$ $\cdot\left(C_{i}^{2}-k_{1} C_{i}\right)=0$. Thus either $p_{1} \neq 0$ or $q_{1} \neq 0$. Now it follows from Lemma 2.5 that the corresponding numbers $\sigma\left(B_{i}\right), \sigma\left(B_{i} B_{i}^{*}\right), \sigma\left(B_{i} C_{i}^{*}\right)$, and $\sigma\left(C_{i}^{*} C_{i} B_{i}\right)$ are equal for $i=1,2$. Thus

$$
\begin{gathered}
p_{1}=p_{2}, \\
\left|q_{1}\right|^{2}+\left|p_{1}\right|^{2}=\left|q_{2}\right|^{2}+\left|p_{2}\right|^{2}, \\
g_{1} q_{1}+k_{2}^{*} p_{1}=g_{2} q_{2}+k_{2}^{*} p_{2}, \\
p_{1}\left(g_{1}^{2}+\left|f_{1}\right|^{2}+\left|k_{2}\right|^{2}\right)+q_{1}\left(d_{1} f_{1}^{*}+k_{1} g_{1}\right) \\
=p_{2}\left(g_{2}^{2}+\left|f_{2}\right|^{2}+\left|k_{2}\right|^{2}\right)+q_{2}\left(d_{2} f_{2}^{*}+k_{1} g_{2}\right) .
\end{gathered}
$$

Also from Lemma 2.4 it follows that

$$
d_{1}^{2}+g_{1}^{2}+\left|f_{1}\right|^{2}=d_{2}^{2}+g_{2}^{2}+\left|f_{2}\right|^{2},
$$




$$
\left|k_{1}-k_{2}\right|^{2} d_{1}^{2}+\left|k_{1}\right|^{2} g_{1}^{2}+d_{1}^{2} g_{1}^{2}=\left|k_{1}-k_{2}\right|^{2} d_{2}^{2}+\left|k_{1}\right|^{2} g_{2}^{2}+d_{2}^{2} g_{2}^{2} \text {. }
$$

Now (1) and (2) yield $\left|q_{1}\right|=\left|q_{2}\right|$, and (1) and (3) yield $g_{1}^{2}\left|q_{1}\right|^{2}=g_{2}^{2}\left|q_{2}\right|^{2}$. Hence if $q_{1} \neq 0$, it follows that $g_{1}=g_{2}$, and subtraction and division in (6) yield $d_{1}=d_{2}$ (remember $k_{1} \neq k_{2}$ ).

Thus from (5), $\left|f_{1}\right|=\left|f_{2}\right|$ and if $g_{1} d_{1}=0, f_{1}=f_{2}$ follows from the hypothesis. If $g_{1} d_{1} \neq 0$, then $f_{1}=f_{2}$ follows from (1). Turning to the case $q_{1}=0, p_{1} \neq 0$, (1) and (4) yield $g_{1}^{2}+\left|f_{1}\right|^{2}=g_{2}^{2}+\left|f_{2}\right|^{2}$, and this with (5) gives $d_{1}=d_{2}$. By subtracting $\left|k_{1}-k_{2}\right|^{2} d_{i}^{2}$ from each side of (6) and dividing the resulting equation by $\left|k_{1}\right|^{2}+d_{i}^{2}$, we get $g_{1}=g_{2}$, and $f_{1}=f_{2}$ follows as above.

TheOREM. If $A_{1}$ and $A_{2}$ are $3 \times 3$ complex matrices, and $T\left(A_{1}\right)=T\left(A_{2}\right)$, then $A_{1}$ is unitarily equivalent to $A_{2}$.

Proof.The traces assumed equal are sufficient to guarantee that $A_{1}$ and $A_{2}$ have the same eigenvalues, and thus the one of the three Lemmas 2.1, 2.2, 2.3 pertaining to $A_{1}$ also pertains to $A_{2}$. Applying the appropriate lemma to the $A_{i}$ yields matrices $B_{i}$ unitarily equivalent to the respective $A_{i}$, and it follows that $T\left(B_{1}\right)=T\left(A_{1}\right)=T\left(A_{2}\right)=T\left(B_{2}\right)$. Now an application of the appropriate Lemmas $2.6,2.7$, or 2.8 completes the argument.

As an immediate corollary we get

Corollary. If $A$ and $B$ are $3 \times 3$ real matrices such that $T(A)=T(B)$, then there is a real orthogonal matrix $U$ satisfying $U A U^{*}=B$.

For a proof of the corollary the reader is referred to [3] where the more general result is demonstrated that any complete set of unitary invariants for complex $n \times n$ matrices is a complete set of orthogonal invariants for real $n \times n$ matrices.

3. In [1] it is claimed that the first six of the nine traces of $T\left(A_{i}\right)$ are a complete set of unitary invariants for $3 \times 3$ matrices $A_{1}$ and $A_{2}$ having distinct eigenvalues. To see that this is not the case, consider matrices $A_{1}$ $=\operatorname{Tri}(0,1,2,1,1,1)$ and $A_{2}=\operatorname{Tri}\left(0,1,2,1 / 3^{1 / 2}, 2^{1 / 2},-2^{1 / 2} / 3^{1 / 2}\right)$. Calculation shows that the first six traces are indeed equal, but also $\sigma\left(A_{1}^{*} A_{1}^{2} A_{1}^{* 2} A_{1}\right)$ $=240$ and $\sigma\left(A_{2}^{*} A_{2}^{2} A_{2}^{* 2} A_{2}\right)=232$, so that $A_{1}$ and $A_{2}$ cannot be unitarily equivalent.

\section{REFERENCES}

1. F. D. Murnaghan, On the unitary invariants of a square matrix, An. Acad. Brasil. Ci. 26 (1954), 1-7. 1942.

2. P. R. Halmos, Finite dimensional vector spaces, Princeton Univ. Press, Princeton, N. J.,

3. C. Pearcy, $A$ complete set of unitary invariants for operators generating finite $W^{*}$-algebras of type I, Pacific J. Math. (to appear).

4. W. Specht, Zur Theorie der Matrizen. II, Jber. Deutsch. Math. Verein. 50 (1940), 19-23.

Rice University,

Houston, Texas 